\title{
Fluorescence Single Particle Tracking for the Characterization of Submicron Protein Aggregates in Biological Fluids and Complex Formulations
}

\author{
Vasco Filipe $\cdot$ Robert Poole $\cdot$ Marika Kutscher $\cdot$ Katrien Forier $\cdot$ Kevin Braeckmans $•$ Wim Jiskoot
}

Received: 1 October 2010 / Accepted: 19 January 2011 / Published online: 5 February 2011

(C) The Author(s) 2011. This article is published with open access at Springerlink.com

\begin{abstract}
Purpose To evaluate the potential of fluorescence single particle tracking (FSPT) for the characterization of submicron protein aggregates in human serum, plasma and formulations containing human serum albumin (HSA).

Methods A monoclonal $\lg G$ was covalently labeled with a fluorescent dye and cross-linked with glutaraldehyde. IgG aggregates and fluorescent beads of $0.1 \mu \mathrm{m}$ (control) were diluted in buffer, serum and plasma, and their size distributions were analyzed by fSPT and nanoparticle tracking analysis (NTA). In a separate experiment, IgG and HSA, fluorescently labeled with different dyes, were mixed and subjected to heat stress. The stressed sample was analyzed by fSPT using a dual color mode and by NTA.
\end{abstract}

Electronic Supplementary Material The online version of this article (doi: | 0. I 007/s | | 095-0 | |-0374-0) contains supplementary material, which is available to authorized users.

V. Filipe $\cdot$ R. Poole $\cdot$ M. Kutscher $\cdot$ W. Jiskoot $(\square)$

Division of Drug Delivery Technology

Leiden/Amsterdam Center for Drug Research

Leiden University

P.O. Box 9502, 2300 RA, Leiden, The Netherlands

e-mail:w.jiskoot@lacdr.leidenuniv.nl

V. Filipe

Department of Pharmaceutics

Utrecht Institute for Pharmaceutical Sciences (UIPS)

Utrecht University

P.O. Box 80082, 3508 TB, Utrecht, The Netherlands

K. Forier $\cdot$ K. Braeckmans

Biophotonic Imaging Group

Laboratory of General Biochemistry \& Physical Pharmacy

Faculty of Pharmacy, Ghent University

Harelbekestraat 72

9000, Ghent, Belgium
Results The accuracy and precision of FSPT proved to be comparable to NTA. fSPT was able to successfully measure all the samples in buffer, serum and plasma. The average size of the cross-linked protein aggregates showed a slight increase in biological fluids. Moreover, fSPT analysis showed that a significant proportion of the aggregates formed by subjecting an $\lg \mathrm{G} / \mathrm{HSA}$ mixture to heat stress were composed of both proteins.

Conclusion fSPT is a powerful technique for the characterization of submicron protein aggregates in biological fluids and complex formulations.

KEY WORDS fluorescence single particle tracking · HSA . $\operatorname{lgG} \cdot$ protein aggregates $\cdot$ serum and plasma

\section{INTRODUCTION}

Over the past few decades, therapeutic proteins have become established as a major drug class for the pharmaceutical industry. A major challenge in commercializing proteins as drug candidates is their inherent physical and chemical instability. Of the numerous degradation pathways, protein aggregation is a particular cause for concern $(1,2)$. It is believed that protein aggregates may have the propensity in vivo to trigger an antibody response against the monomeric form of that protein, also known as unwanted immunogenicity $(1,3)$. The presence of antibodies against a therapeutic protein can have serious clinical consequences, such as loss of therapeutic efficacy or even the neutralization of the equivalent endogenous protein (4). Therefore, determination of the amount and type of aggregates necessary to allegedly trigger such immune reactions is of major importance both for pharmaceutical companies and regulatory agencies. With regard to their size, protein- 
containing samples can be very heterogeneous, and individual aggregates can range in diameter from a few nanometers to several micrometers or larger $(2,5,6)$.

A number of characterization tools are available for determining the size of protein aggregates, e.g. size exclusion chromatography and asymmetrical flow field-flow fractionation combined with UV and light scattering detectors, dynamic light scattering (DLS) and nanoparticle tracking analysis (NTA), analytical ultracentrifugation, light obscuration particle counting and micro flow imaging (7-11). A major limitation of most of these techniques is that it is very difficult, if not impossible, to use them for analyzing therapeutic proteins or their aggregates in biological media. In fact, very little is known about the fate of protein pharmaceuticals following administration to a patient. To address this problem, we set out to identify a new strategy and analytical tool that would allow us to characterize aggregates of a specific protein in the presence of serum or plasma.

Very recently, fluorescence Single Particle Tracking (fSPT) was suggested as a powerful method to size submicron matter in undiluted biological fluids (12). Using fSPT, it was shown that the aggregation of fluorescently labeled liposomal drug carriers can be followed in real time in undiluted whole blood. In the present work, we have evaluated the potential of the ASPT technique to detect and determine the size of submicron protein aggregates in serum and plasma. To this end, we have covalently labeled the proteins of interest with a fluorescent probe. This strategy has previously been applied for the characterization of a specific protein interaction in human serum using analytical ultracentrifugation in an instrument modified to incorporate a fluorescence detection system (13). The authors demonstrated that the fluorescence probe did not affect either the characteristics or the potency of the protein. The same labeling strategy may also be used to characterize the aggregates of therapeutic proteins in complex formulations containing, for example, high amounts of stabilizing proteins, such as human serum albumin (HSA).

In this work, a monoclonal antibody (MAb) was fluorescently labeled without affecting its aggregation profile. Submicron MAb aggregates were generated by applying stress and were successfully analyzed in biological fluids and in a formulation containing a large amount of HSA.

\section{MATERIAL AND METHODS}

\section{Diluents}

The formulation buffer used to prepare the polystyrene beads and proteins contained $10 \mathrm{mM}$ sodium citrate
(Merck, Darmstadt, Germany), 5\% (w/v) sucrose (SigmaAldrich, Buchs, Switzerland), $\mathrm{pH}$ 6.0. The formulation buffer was filtered using a $0.22-\mu \mathrm{m}$ PES low binding syringe-driven filter unit (Millex ${ }^{\mathrm{TM}} \mathrm{GP}$, Millipore, Ireland).

Human serum and plasma were collected from three healthy volunteers free of medications. Serum was collected in Vacutainer SST tubes, and plasma was collected in Vacutainer heparin tubes (Becton Dickinson, Franklin Lakes, USA). The plasma samples were centrifuged for $2 \mathrm{~min}$ at 2,000 rpm with a Sigma 1-15 centrifuge (Osterode, Germany) mainly to spin down the red blood cells. The serum samples were centrifuged for $15 \mathrm{~min}$ at 3,000 rpm in a Beckman Coulter Alegra X-12 centrifuge (Brea, USA) to remove all the blood cells and clotting factors. The serum and plasma samples were stored at $4^{\circ} \mathrm{C}$ for a maximum period of $48 \mathrm{~h}$ before being used for measurements. The viscosities of the buffer, serum and plasma were measured in an AR-G2 rheometer from TA Instruments (New Castle, USA) at $37^{\circ} \mathrm{C}$. The average values used for sizing calculations are $0.80 \mathrm{cP}, 1.29 \mathrm{cP}$ and $1.30 \mathrm{cP}$ for buffer, serum and plasma, respectively.

\section{Preparation of Fluorescent Polystyrene Beads}

Polystyrene FluoSpheres carboxylate-modified microspheres with a nominal size of $0.1 \mu \mathrm{m}$, yellow-green fluorescent (excitation $505 \mathrm{~nm} /$ emission $515 \mathrm{~nm}$ ), were purchased from Invitrogen (Merelbeke, Belgium) and covalently linked with methoxy-polyethylene glycol-amine (m-PEG-amine) (5000 Da, Creative PEGWorks, Winston Salem, U.S.A.) polymer chains using a method adapted from Suh et al. in order to minimize interactions with serum components (14). The fluorescent PEGylated beads were separately diluted with formulation buffer, serum and plasma until the concentration was acceptable for either NTA or fSPT measurements, i.e. 1:5,000 volume-based dilution for NTA measurements and 1:1,000 volume-based dilution for ISPT measurements.

\section{Preparation of Alexa Fluor-Labeled IgG}

Alexa Fluor 488, Alexa Fluor 546 and Alexa Fluor 594 carboxylic acid, N-hydroxysuccinimide esters were obtained from Invitrogen (Merelbeke, Belgium). IgG and HSA labeling was performed according to the manufacturer's instructions. In brief, a recombinant human MAb of the $\operatorname{IgG}_{1}$ subclass (11) was prepared at a concentration of $10 \mathrm{mg} / \mathrm{ml}$ in $100 \mathrm{mM}$ sodium phosphate (Fluka, Sigma-Aldrich, Buchs, Switzerland) $\mathrm{pH}$ 7.4. To this was added a solution of dye in DMSO $(10 \mathrm{mg} / \mathrm{ml})$ to give a molar ratio of 4:1 (dye:protein). The same procedure was followed for HSA. A pH of 7.4 was chosen in order to achieve selective labeling of the amine termini. 
The samples were mixed and left for $1 \mathrm{~h}$ at room temperature. The conjugates were purified by passing the samples through disposable PD10 desalting columns (GE Healthcare, Uppsala, Sweden) packed with Sephadex G25 medium that had been equilibrated with phosphatebuffered saline (PBS). Finally, the samples were dialyzed using a Float-A-Lyzer ${ }^{\circledR}$ G2 (Spectrum, Rancho Dominguez, USA) with a $100 \mathrm{kDa}$ molecular weight cut-off membrane to remove excess dye and to exchange the buffer from PBS back to the original formulation buffer (see Diluents subsection). The final labeling ratio achieved was about four Alexa Fluor 546 labels per IgG, about two Alexa Fluor 594 labels per IgG and about two Alexa Fluor 488 labels per HSA, according to the formula suggested by Invitrogen:

$\frac{n_{\text {Dye }}}{n_{\text {Protein }}}=\frac{A_{\max }}{\varepsilon_{\text {Dye }} \times c_{\text {Protein }} \times l}$

where $n_{\text {Dye }}$ is the moles of dye, $n_{\text {Protein }}$ is the moles of protein, $A_{\max }$ is the measured absorbance at the dye's absorption maximum, $\varepsilon_{\text {dye }}$ is the molar extinction coefficient of the dye at its absorption maximum, $c_{\text {Protein }}$ is the molar protein concentration (determined by absorbance at 280 and corrected for the presence of dye, according to the correction formulas given by the dye supplier) and $l$ is the path length.

\section{Preparation of Fluorescently Labeled Protein Aggregates}

Covalent aggregates of Alexa Fluor 546-labeled IgG (A546 IgG) were prepared by using glutaraldehyde (Sigma-Aldrich, Steinheim, Germany) as a cross-linker. The aggregates were obtained by incubating $0.5 \mathrm{mg} / \mathrm{ml}$ of A546 IgG with $0.2 \%(\mathrm{v} / \mathrm{v})$ of glutaraldehyde for $2 \mathrm{~min}$ at room temperature. The cross-linking reaction was terminated by adding sodium borohydride (SigmaAldrich, Steinheim, Germany) at a final concentration of $0.008 \%(\mathrm{w} / \mathrm{v})$. This reaction was then allowed to proceed for $24 \mathrm{~h}$ at room temperature. The sample was diluted 250-fold with the formulation buffer, serum or plasma before the fSPT analysis and 5-fold before the NTA measurements.

For the preparation of fluorescently labeled protein aggregates in a complex formulation, Alexa Fluor 594labeled $\operatorname{IgG}$ (A594 IgG) was prepared at a concentration of $0.5 \mathrm{mg} / \mathrm{ml}$ in formulation buffer (see Diluents subsection) containing Alexa Fluor 488-labeled HSA (A488 HSA) at a concentration of $3.2 \mathrm{mg} / \mathrm{ml}$. The sample was placed in a 1.5-ml reaction tube (Eppendorf, Hamburg, Germany) and incubated for $12 \mathrm{~min}$ at $74^{\circ} \mathrm{C}$ in a VWR Digital Heat Block (VWR, West Chester, USA). The sample was diluted 1,000-fold with the formulation buffer before the ISPT and 50-fold before the NTA measurements.

\section{Nanoparticle Tracking Analysis (NTA)}

NTA measurements were performed essentially as described before with a NanoSight LM20 (NanoSight, Amesbury, United Kingdom), equipped with a sample chamber with a 640-nm laser and a Viton fluoroelastomer O-ring (11). The samples were injected in the sample chamber with sterile syringes (BD Discardit II, New Jersey, USA), and all measurements were performed at $37^{\circ} \mathrm{C}$. The software used for capturing and analyzing the data was the NTA 2.0 Build 127. The samples were measured for $40 \mathrm{~s}$ with manual shutter and gain adjustments. Three measurements of the same sample were performed for the polystyrene beads and six measurements for the protein aggregates. The standard deviations of the mean size values were calculated from the values obtained in each measurement.

\section{Light Obscuration (LO)}

LO measurements were performed on a PAMAS SVSS system (PAMAS GmbH, Rutesheim, Germany) equipped with a HCB-LD-25/25 sensor and a $1 \mathrm{ml}$ syringe. Each sample was measured three times, with each measurement consisting of a pre-run volume of $0.3 \mathrm{ml}$ followed by three runs of $0.2 \mathrm{ml}$ at a flow rate of $10 \mathrm{ml} / \mathrm{min}$. The measurement settings differ significantly from the United States Pharmacopeia (USP) method, but they were chosen in order to reduce sample volume, since it has been shown that small sampling volumes are appropriate for quantifying size and levels of subvisible particles at the amounts typically present in protein therapeutics (15). The samples were diluted 100-fold with the formulation buffer before the measurements in order to keep the particle counts below the upper count limit of the device.

\section{Fluorescence Single Particle Tracking (fSPT)}

The fSPT technique was recently described in detail elsewhere (12). In short, widefield laser illumination was provided to an inverted epi-fluorescence microscope (Nikon TE2000E, NIKON BELUX, Brussels, Belgium) for excitation of the fluorescently labeled sample. The fSPT setup was equipped with three solid state lasers: a 100 mW Calypso 491 nm (Cobolt, Solna, Sweden), a $75 \mathrm{~mW}$ Jive $561 \mathrm{~nm}$ (Cobolt) and a IQ1C $30 \mathrm{~mW} 636 \mathrm{~nm}$ (Power Technology, Little Rock, AR). Using a fast and sensitive electron-multiplying CCD camera (Cascade II:512; Roper Scientific, Tucson, AZ), videos were acquired (Nikon Elements $\mathrm{R}$ imaging software) of the fluorescently labeled protein aggregates diffusing in the 
medium (formulation buffer, serum, or plasma). A Nikon Plan Apochromat $100 \times$ NA1.4 oil immersion objective lens was used for imaging.

Using custom-built software, diffusional motion trajectories were calculated for individual protein aggregates from the fSPT videos. Since the diffusion coefficient for each trajectory could be calculated, a distribution of diffusion coefficients was obtained by analyzing a large number of trajectories. A maximum entropy deconvolution algorithm was used to reduce statistical broadening and sampling noise in the distribution of diffusion coefficients. Finally, this distribution of diffusion coefficients was converted to a size distribution using the Stokes-Einstein equation for spherical particles:

$d=\frac{k T}{3 D \pi \eta}$

where $d$ is the particle diameter, $k$ the Boltzmann constant, $\mathcal{T}$ the absolute temperature, $\eta$ the dynamic viscosity of the solution and $D$ the diffusion coefficient.

Each measurement consisted of the recording and analysis of ten videos of the same sample for the polystyrene beads and 20 videos for the protein aggregates. Each video was recorded for only $5 \mathrm{~s}$ in order to minimize photobleaching. The standard deviations of the mean size values were calculated from the values obtained in each video.

The upper and lower size and concentration limits of fSPT are hard to specify, since they are dependent on several complex analytical factors. The lower size limit for detecting a particle depends on the ratio between the background intensity and the number of photons that can be collected from that particle, which in turn is dependent on the brightness of the fluorescent label, laser intensity and illumination time. Nevertheless, in our experiments, particles as small as $50 \mathrm{~nm}$ could be successfully measured. The upper size limit is mostly related to the particle density and fluid viscosity in such a way that particle sedimentation becomes the limiting factor. In a general way, an upper size limit of about $1000 \mathrm{~nm}$ can be set for fSTP measurements.

With this technique, the upper limit of particle concentration is dictated by the optical resolution and the tracking algorithm, resulting in a maximum useful particle concentration that is typically in the nanomolar range (12). Theoretically, there is no lower limit of particle concentration, since the size is calculated for individual particles. However, it is clear that there should be a sufficient number of particles in the system to obtain good statistics within a reasonable time period.

\section{Colocalization Analysis}

In order to verify whether IgG and HSA can form mixed aggregates, A594 IgG (red fluorescent) and A488 HSA (green fluorescent) were combined, and the mixture was subsequently subjected to heat stress $\left(12 \mathrm{~min}\right.$ at $\left.74^{\circ} \mathrm{C}\right)$. The resulting aggregate-containing solution was then analyzed by dual color $\mathrm{ESPT}$.

Dual color is an fSPT mode in which videos can be recorded simultaneously in two different spectral ranges (green and red in this case), enabling separate video analysis and the detection of particle colocalization between both videos. Since aggregates are seen in $\mathrm{ASPT}$ videos as separate objects (spots of light), an object-based algorithm was employed for quantification. For each frame of the movie, all green and red objects were identified by image processing (for details, see Braeckmans et al.) (16). For each object, the contour and centre position were determined. A green object was considered colocalized with a red object if its center position fell within the contour of the red object. Vice versa, a red object was considered colocalized with a green object if its center position fell within the contour of the green object. By performing this analysis for all frames of a movie, the percentage of mixed aggregates relative to the pure IgG or HSA aggregates could be estimated.

\section{RESULTS AND DISCUSSION}

\section{Fluorescent Polystyrene Beads in Biological Fluids}

In line with previous studies, we first verified that fSPT can accurately detect and size submicron particles in biological fluids (12). PEGylated standard fluorescent polystyrene beads of $0.1 \mu \mathrm{m}$ nominal size were separately diluted in buffer, serum and plasma, and the size distributions were then measured by fSPT (Fig. 1). The samples in buffer were also analyzed by NTA as a control, since both sizing techniques are based on the same principle of tracking individual particles and calculating the particle hydrodynamic size from the measured diffusion coefficient. However, the standard NTA instrument, as described by Filipe et al. (11), relies on light scattered by the particles and, therefore, cannot be used for biological media like serum or plasma, which contain a variety of light scattering components.

The size distributions obtained by fSPT and NTA for the PEGylated beads in buffer are comparable, confirming the accuracy of ASPT to size nanoparticles. The mean size given by NTA was $129 \pm 7 \mathrm{~nm}$, while the mean sizes given by ASPT were $139 \pm 10 \mathrm{~nm}$ in buffer, $143 \pm 7 \mathrm{~nm}$ in serum and $142 \pm 8 \mathrm{~nm}$ in plasma. The source of the small difference between the means given by the two techniques in buffer is unknown, but may have to do with the contribution of small impurities (between 50 and $120 \mathrm{~nm}$ ) associated with the PEGylation process. Such impurities would decrease the NTA mean but cannot be detected by 


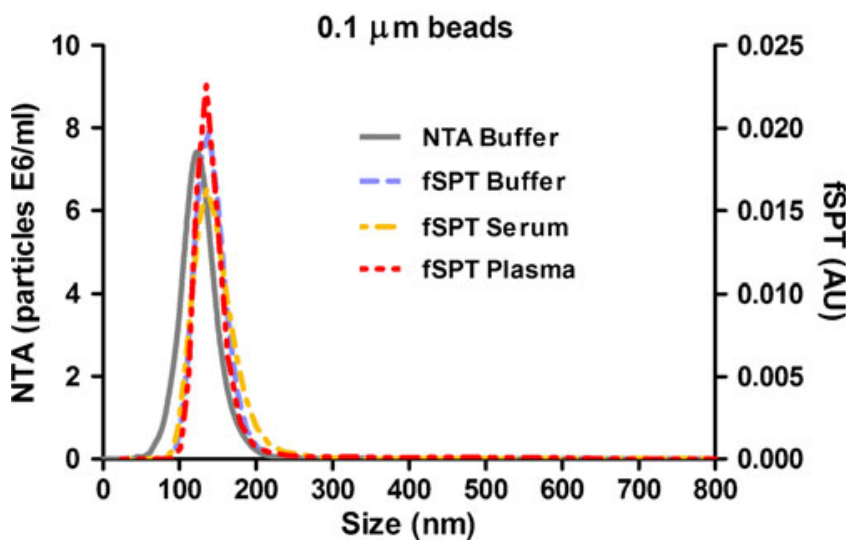

Fig. I Size distribution from fSPT and NTA measurements of PEGylated fluorescent polystyrene beads in buffer, serum and plasma.

fSPT, since they would not be fluorescent. In any case, the mean values are within the standard deviations obtained for both techniques. These standard deviations of the means are small and very similar between the two techniques, confirming the precision of fSPT.

The fSPT size distributions are also very similar in each of the media, indicating that the beads do not aggregate in biological fluids and that these fluids do not interfere with the fluorescence properties of the beads. Moreover, it can be concluded that the clotting factors, which are present in plasma and not in serum, have no major effect on the size distribution of the beads. These results demonstrate the potential of this technique to measure the size distribution of nanoparticles in biological fluids.

\section{Effect of Labeling on Aggregation Profile}

With the aim of evaluating the effect of the fluorescent label on the aggregation profile of this specific $\mathrm{IgG}$, a comparative aggregation study between A546-labeled and unlabeled IgG was performed. The samples were stressed by glutaraldehyde cross-linking under the same conditions, and the aggregates were analyzed by NTA and LO (Fig. 2). Combined, these two techniques focus on the size range covered by fSPT (submicron range) and on the size range that can interfere with fSPT measurements (micron range).

The NTA size distribution of the labeled IgG samples is very similar to that of the unlabeled one. The height difference between the two peaks is within the variations inherent to the stress method itself and therefore should not be taken into account. The LO measurements also show a similar size distribution and particle count per size bin between the two samples. These results lead to the conclusion that this fluorescent label does not have a major impact on the aggregation profile in the subvisible and submicron range of this specific IgG with this particular stress method.

These findings should not be generalized for other proteins, other fluorescent labels or other stress methods. During optimization studies, we verified that some fluorescent dyes may significantly change the aggregation profile of some proteins in certain given conditions (data not shown). Therefore, a case-by-case approach should be followed to evaluate the effect of a fluorescent label on a certain protein's stability.

\section{Covalent A546 IgG Aggregates in Biological Fluids}

In order to evaluate the analytical performance of ASPT for protein aggregates in biological fluids, covalent submicron A546 IgG aggregates were analyzed in buffer, serum and plasma (Fig. 3). Glutaraldehyde cross-linked covalent aggregates were used, since they cannot be dissociated and can be considered to be stable aggregates. Unstressed A546 IgG was also analyzed by fSPT in each of the media and was shown not to contain aggregates. As an additional control, the sample diluted in buffer was also measured by NTA.
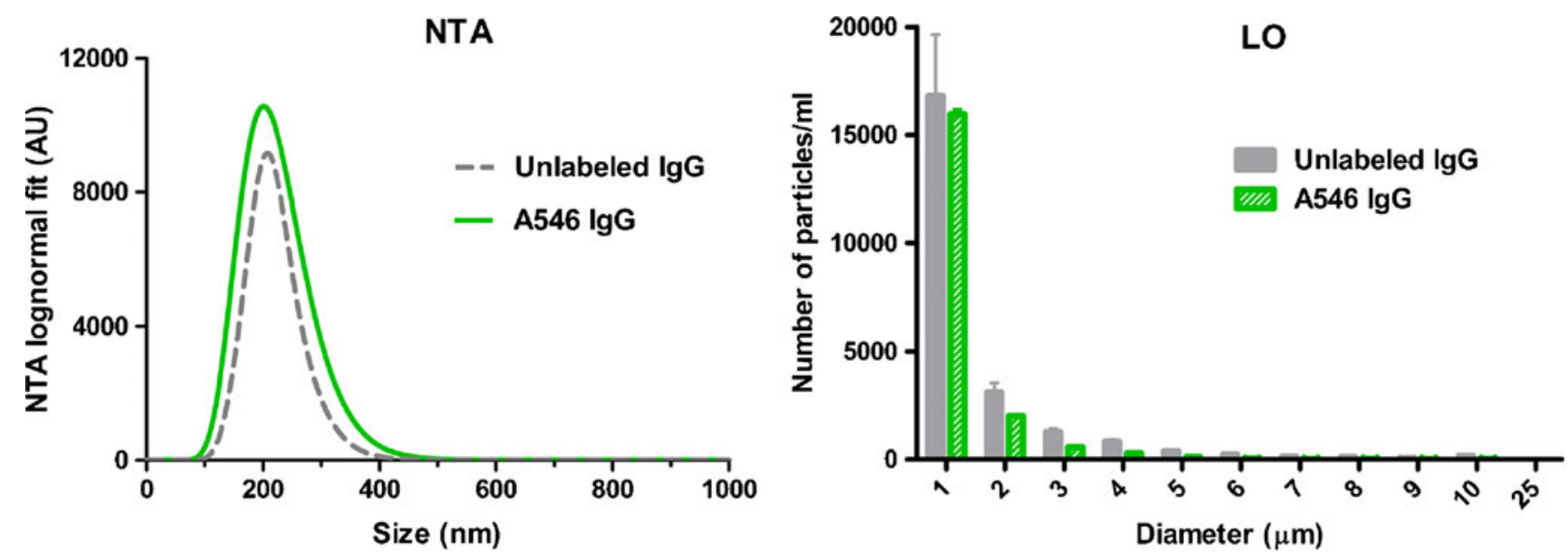

Fig. 2 Size distribution from NTA and LO measurements in buffer of A546 lgG and unlabeled lgG aggregates induced by glutaraldehyde cross-linking. 


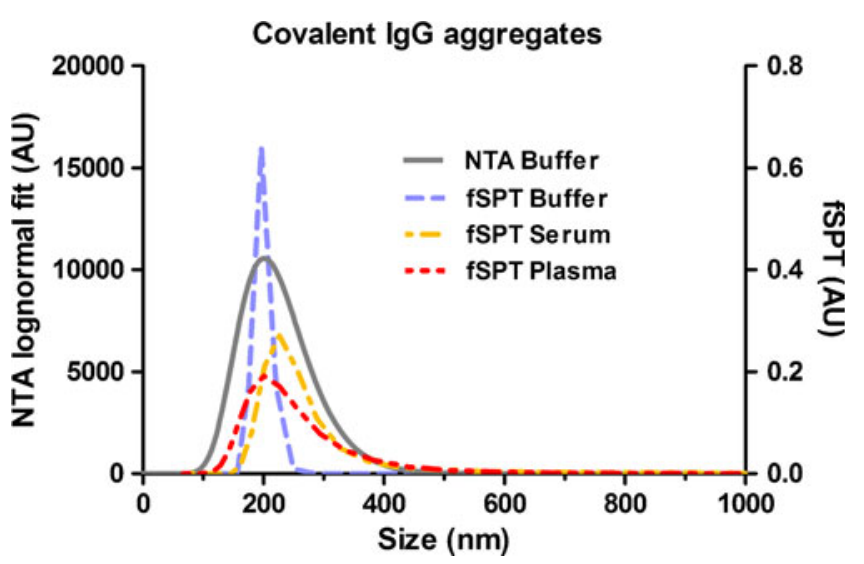

Fig. 3 Size distribution from fSPT and NTA measurements of glutaraldehyde cross-linked A546 lgG in buffer, serum and plasma.

The size average of the A546 IgG aggregates in buffer measured with NTA was $215 \pm 38 \mathrm{~nm}$ and with fSPT $198 \pm$ $36 \mathrm{~nm}$. The difference observed in peak width between the NTA and fSPT size distributions in buffer can be attributed to a difference in the size analysis algorithm. In the fSPT sizing method used here, the raw size distribution as measured directly from the particle trajectories is refined by a maximum entropy deconvolution step that filters out statistical broadening and sampling noise so as to not overinterpret features in the distribution that are not statistically warranted by the data (12). Although a similar function is available in the NTA software (model curve fitting), we chose to use the log-normal fit of the data for this sample, given that according to the manufacturer, this function should only be used for either very monodisperse or clearly bimodal samples. Another factor that can contribute to the difference in peak width is the difference in optimal dilutions (50-fold) required for measurements with each technique, since higher dilutions may affect the aggregate size distribution (7).

These glutaraldehyde cross-linked aggregates showed an increase in their mean size in serum and plasma compared to buffer $(198 \pm 36 \mathrm{~nm}, 265 \pm 38 \mathrm{~nm}$ and $245 \pm 34 \mathrm{~nm}$ in buffer, serum and plasma, respectively). This size increase could be due to self-association of the aggregates or to interactions between the aggregates and other serum and plasma components, a phenomenon normally described as opsonization (17). In general, opsonization is the adsorption of plasma components (i.e. opsonins) onto the surface of a foreign particle to render it more susceptible to phagocytosis. Opsonins include mainly immunoglobulins, blood clotting factors and components of the complement system $(18,19)$. Given that the $\operatorname{IgG}$ used in this work is humanized and that $\mathrm{IgG}$ is one of the most abundant classes of proteins circulating in blood, one would not expect it to be opsonized. However, protein cross-linking with glutaraldehyde leads to a high amount of intermolecular and intramolecular cross-links, which normally lead to conformational changes (20-22). Consequently, since opsonins bind to nanoparticles mainly via hydrophobic interactions, it is possible that these unnatural aggregates of conformationally changed IgG may lead to some opsonization (23).

Given that the size distribution of these aggregates is relatively the same in serum and in plasma, it is possible to conclude that the presence of clotting factors has little impact on the hypothetical opsonization, since they do not have a major effect on the size average of these aggregates.

\section{A594 IgG Aggregates Formulated with A488 HSA}

Over the last few decades, the need to stabilize therapeutic proteins progressively led to rather complex formulations containing sugars, surfactants, amino acids and even other proteins. However, such intricate environments often make the selective characterization of the therapeutic protein very difficult. For example, several therapeutic protein formulations contain high concentrations of HSA, which is known to act as a general stabilizer for liquid and lyophilized protein formulations by effectively inhibiting protein surface adsorption $(24,25)$. Unfortunately, with most protein characterization tools, it is virtually impossible to distinguish between the excipient HSA and the therapeutic protein without prior separation. When considering protein aggregates, the situation becomes even more complex, as both the protein drug and HSA may form separate or mixed aggregates.

This section aims to show the potential of fSPT for the selective characterization of protein aggregates in formulations containing high amounts of HSA. For this purpose, A594 IgG was mixed with A488 HSA at an IgG:HSA concentration ratio of 1:6 $(\mathrm{w} / \mathrm{w})$; the mixture was heated in order to stimulate the formation of aggregates with diameters of the order of a hundred nanometers (monitored by fSPT). The sample was then analyzed by fSPT using dual color imaging (Fig. 4), whereby Alexa Fluor 488 and Alexa

\section{Colocalization of IgG and HSA}

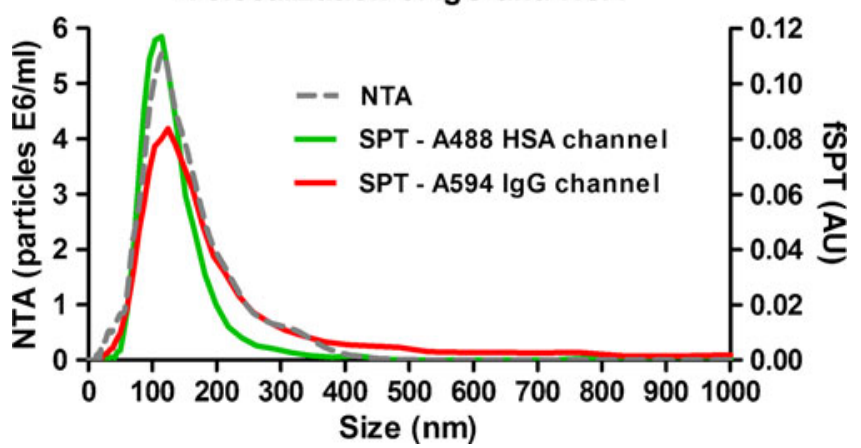

Fig. 4 Size distribution from fSPT and NTA measurements of a heatstressed mixture of A594 $\lg G$ and A488 HSA in buffer. 
Table I Mean Size from NTA and fSPT Measurements of a HeatStressed Mixture of A594 lgG and A488 HSA in Buffer

\begin{tabular}{llll}
\hline & fSPT & & NTA \\
\cline { 2 - 3 } & Mean (nm) & Colocalization (\%) & Mean (nm) \\
\hline Scattering & - & - & I52 \\
A488 HSA Channel & I22 & $36 \pm 4$ & - \\
A594 IgG Channel & 161 & $70 \pm 9$ & - \\
\hline
\end{tabular}

Fluor 594-labeled aggregates could be tracked simultaneously to examine whether HSA and IgG form mixed aggregates or not. The overall aggregate size was also measured by NTA, although it cannot distinguish between IgG aggregates, HSA aggregates or mixed aggregates (Fig. 4).

The average size of the aggregates determined by NTA was $152 \mathrm{~nm}$. By fSPT, the average size of the IgGcontaining and HSA-containing aggregates was $161 \mathrm{~nm}$ and $122 \mathrm{~nm}$, respectively (Table I). These results suggest that either the aggregates of both proteins have coincidently the same size range or that these aggregates are composed of both proteins. In order to clarify this uncertainty, the videos from both IgG (red fluorescence) and HSA (green fluorescence) channels were aligned, and an object-based colocalization was calculated. A representative ISPT video frame is shown on Fig. 5, wherein colocalization is clearly visible (see also Supplementary Material for complete overlaid video). The results show that the IgG-containing aggregates colocalize significantly with the HSA-containing aggregates. About $70 \pm 9 \%$ of the aggregates tracked in the IgG channel were found to be colocalized with HSAcontaining aggregates, while only about $36 \pm 4 \%$ of the aggregates tracked in the HSA channel colocalized with IgG-containing aggregates. The amount of aggregates tracked in the HSA channel was approximately twice the amount of aggregates tracked in the IgG channel. This indicates that there were more HSA-containing aggregates than IgG-containing aggregates, and this explains the low colocalization observed for the aggregates in the HSA channel. The fact that there were more HSA aggregates is not surprising considering the IgG:HSA concentration ratio of 1:6 (w/w). An example of this occurrence can be seen in Fig. 5, where two out of five HSA aggregates do not colocalize with IgG aggregates.

Previous studies have already suggested the interaction between therapeutic proteins and HSA to form mixed aggregates in solution, mainly by enzyme-linked immunosorbent assays $(26,27)$. In the present study, using dual color ASPT, we were for the first time able to visualize and get a clear glimpse at the aggregation profile of therapeutic proteins in formulations containing HSA. Here, we have clearly demonstrated that under conditions of heat stress, HSA can form mixed aggregates with the therapeutic protein: in this case, IgG. Given that heat stress induces protein denaturation and consequently exposure of hydrophobic regions, it is likely that HSA starts to bind to the partially denatured intermediates of the therapeutic protein and ends up involved in the overall aggregation process $(25,28)$. In fact, the same heat stress was applied to the A594 IgG in a formulation without A488 HSA, and it resulted in a much higher amount of IgG aggregates with a higher size average, confirming the stabilizing properties of HSA in solution (data not shown).

It should be noted that control experiments in buffer with only one of the two proteins labeled indicated that the labeling of HSA or IgG did not change the size distribution. However, labeling of HSA (but not $\operatorname{IgG}$ ) substantially reduced the amount of submicron aggregates (as measured by NTA), even though the amount of micron-sized aggregates (as measured by LO) remained approximately the same (data not shown). This may be due to the hydrophobic nature of the label and/or the (random) HSA labeling procedure. Further studies with other labels or different, site-specific labeling strategies should be done in order to understand and avoid this problem.
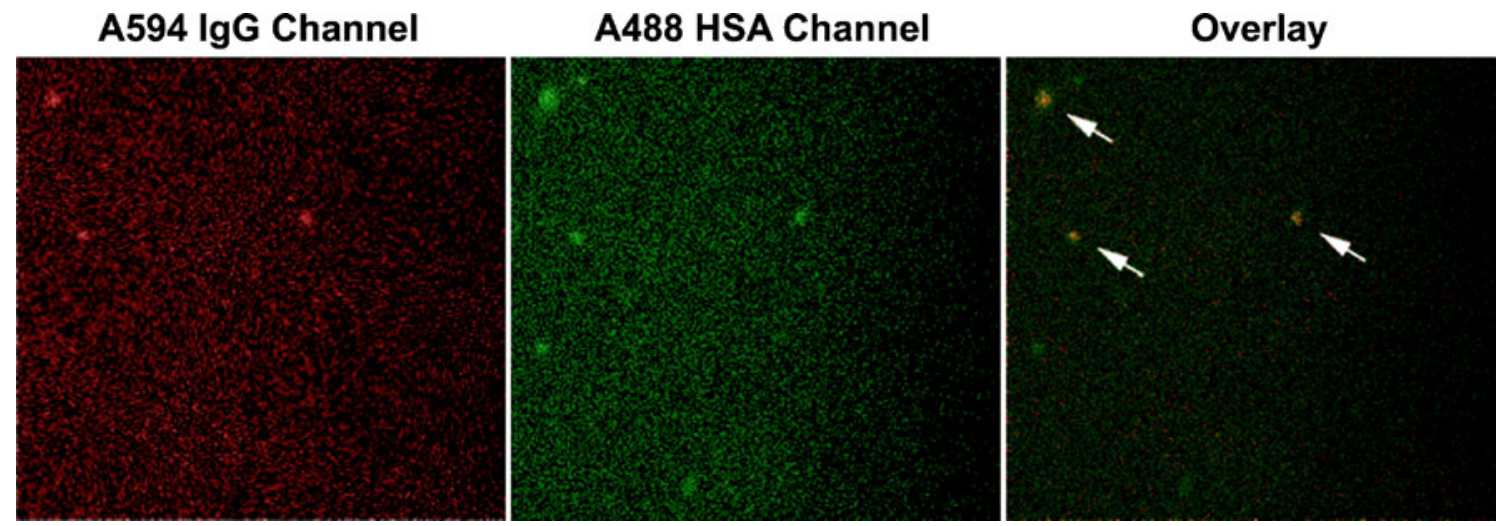

Fig. 5 A video frame containing aggregates captured in the $\lg G$ and HSA channels using the dual color mode of fSPT, with the corresponding overlay image. 


\section{CONCLUDING REMARKS}

In this work, we evaluated the potential of fSPT for the characterization of protein aggregates in biological fluids and HSA-containing formulations. ASPT gave comparable size distributions to NTA for aggregates and particles in buffer and has the added advantage that it can also be applied to look at samples in serum and plasma. It seems that some serum components adsorb to the beads and protein aggregates once these come into contact with biological fluids. FSPT has also been shown to be suitable for selectively analyzing protein aggregates in complex formulations, such as those containing HSA. Using dual color fSPT, it was possible to verify that most of the IgG aggregates created by heat stress in an HSAcontaining formulation were in fact composed of a mixture of IgG and HSA.

The requirement for prior labeling of the protein of interest with a fluorescent probe may be seen as a drawback of this technique. However, if it can be proven that the stability and aggregation profile of the proteins remain unaltered after the labeling, the potential of this technique to reveal the fate of protein aggregates in biological fluids is evident. This paves the way for several research areas, such as formulation and immunogenicity studies, where it is important to know if a certain therapeutic protein aggregates once injected into the bloodstream or if certain types of aggregates disappear or change their size in biological fluids.

\section{AKNOWLEDGMENTS}

This research is supported by the Dutch Technology Foundation STW, applied science division of NWO and the Technology Program of the Ministry of Economic Affairs. The authors are grateful to Mies van Steenbergen from the Utrecht Institute for Pharmaceutical Sciences for the viscosity measurements of all the diluents.

Open Access This article is distributed under the terms of the Creative Commons Attribution Noncommercial License which permits any noncommercial use, distribution, and reproduction in any medium, provided the original author(s) and source are credited.

\section{REFERENCES}

1. Cromwell ME, Hilario E, Jacobson F. Protein aggregation and bioprocessing. AAPS J. 2006;8:E572-9.

2. Mahler HC, Friess W, Grauschopf U, Kiese S. Protein aggregation: pathways, induction factors and analysis. J Pharm Sci. 2009;98:2909-34.

3. Rosenberg AS. Effects of protein aggregates: an immunologic perspective. AAPS J. 2006;8:E501-7.
4. Schellekens H. Bioequivalence and the immunogenicity of biopharmaceuticals. Nat Rev Drug Discov. 2002;1:457-62.

5. Hawe A, Kasper JC, Friess W, Jiskoot W. Structural properties of monoclonal antibody aggregates induced by freeze-thawing and thermal stress. Eur J Pharm Sci. 2009;38:79-87.

6. Carpenter JF, Randolph TW, Jiskoot W, Crommelin DJ, Middaugh CR, Winter G, et al. Overlooking subvisible particles in therapeutic protein products: gaps that may compromise product quality. J Pharm Sci. 2009;98:1201-5.

7. Philo JS. A critical review of methods for size characterization of non-particulate protein aggregates. Curr Pharm Biotechnol. 2009;10:359-72.

8. Huang CT, Sharma D, Oma P, Krishnamurthy R. Quantitation of protein particles in parenteral solutions using micro-flow imaging. J Pharm Sci. 2009;98:3058-71.

9. Fraunhofer W, Winter G. The use of asymmetrical flow field-flow fractionation in pharmaceutics and biopharmaceutics. Eur J Pharm Biopharm. 2004;58:369-83.

10. Arakawa T, Wen TJ. Size-exclusion chromatography with on-line light scattering. Curr Protoc Protein Sci. 2001 Chapter 20:Unit 2026.

11. Filipe V, Hawe A, Jiskoot W. Critical evaluation of nanoparticle tracking analysis (NTA) by NanoSight for the measurement of nanoparticles and protein aggregates. Pharm Res. 2010;27:796810.

12. Braeckmans K, Buyens K, Bouquet-Geerardyn W, Vervaet C, Joye P, De Vos F, et al. Sizing nanomatter in biological fluids by fluorescence Single Particle Tracking. Nano Lett. 2010;10:443542.

13. Demeule B, Shire SJ, Liu J. A therapeutic antibody and its antigen form different complexes in serum than in phosphatebuffered saline: a study by analytical ultracentrifugation. Anal Biochem. 2009;388:279-87.

14. Suh J, Choy KL, Lai SK, Suk JS, Tang BC, Prabhu S, et al. PEGylation of nanoparticles improves their cytoplasmic transport. Int J Nanomed. 2007;2:735-41.

15. Cao S, Jiang Y, Narhi L. A light-obscuration method specific for quantifying subvisible particles in protein therapeutics. Pharmacop Forum. 2010;36:824-34.

16. Braeckmans K, Vercauteren D, Demeester J, De Smedt SC. Single particle tracking. In: Diaspro A, editor. Nanoscopy multidimensional optical fluorescence microscopy. Taylor and Francis. 2010.

17. Yoo JW, Chambers E, Mitragotri S. Factors that control the circulation time of nanoparticles in blood: challenges, solutions and future prospects. Curr Pharm Des. 2010;16:2298-307.

18. Moghimi SM, Hunter AC, Murray JC. Long-circulating and target-specific nanoparticles: theory to practice. Pharmacol Rev. 2001;53:283-318.

19. Frank MM, Fries LF. The role of complement in inflammation and phagocytosis. Immunol Today. 1991;12:322-6.

20. Cheung DT, Nimni ME. Mechanism of crosslinking of proteins by glutaraldehyde II. Reaction with monomeric and polymeric collagen. Connect Tissue Res. 1982;10:201-16.

21. Usha R, Ramasami T. Structure and conformation of intramolecularly cross-linked collagen. Colloids Surf B Biointerfaces. 2005;41:21-4.

22. Hermeling S, Aranha L, Damen JM, Slijper M, Schellekens H, Crommelin DJ, et al. Structural characterization and immunogenicity in wild-type and immune tolerant mice of degraded recombinant human interferon alpha2b. Pharm Res. 2005;22:1997-2006.

23. Gessner A, Waicz R, Lieske A, Paulke B, Mader K, Muller RH. Nanoparticles with decreasing surface hydrophobicities: influence on plasma protein adsorption. Int J Pharm. 2000;196:245-9.

24. Wang W. Lyophilization and development of solid protein pharmaceuticals. Int J Pharm. 2000;203:1-60. 
25. McGoff P, Scher DS. Solution formulation of proteins/peptides. In: McNally EJ, Hastedt JE, editors. Protein formulation and delivery, vol. 175. New York: Informa Healthcare USA, Inc.; 2000.

26. Braun A, Alsenz J. Development and use of enzyme-linked immunosorbent assays (ELISA) for the detection of protein aggregates in interferon-alpha (IFN-alpha) formulations. Pharm Res. 1997;14:1394-400.
27. Kumarasamy R, Bausch J, Kopcha D, Patel S, McGonigle E. An enzyme-linked immunosorbent assay (ELISA) for quantitation of adducts of granulocyte-macrophage colony stimulating factor (GM-CSF) and human serum albumin (HSA) in stressed solution mixtures. Pharm Res. 1994;11:365-71.

28. Wang W, Nema S, Teagarden D. Protein aggregation-Pathways and influencing factors. Int J Pharm. 2010;390:89-99. 